Int. J. Morphol.,

32(2):488-492, 2014

\title{
Análisis Morfométrico de Hepatoesteatosis Microvesicular Alcohólica de Ratas
}

\author{
Morphometric Analysis of Alcoholic Microvesicular Hepatic Steatosis in Rats
}

R. Cornejo*; O. Garrido**; L. Sáez*; C. Bustamante* \& G. Cartes*

CORNEJO, R.; GARRIDO, O.; SÁEZ, L.; BUSTAMANTE, C. \& CARTES, G. Análisis morfométrico de hepatoesteatosis microvesicular alcohólica de ratas. Int. J. Morphol., 32(2):488-492, 2014.

RESUMEN: Un total de 24 ratas hembras de 4 meses de vida con peso aproximado de 250 gramos fueron divididas en dos grupos de animales rotulados como A y B. El grupo A se mantuvo con pellet y agua ad libitum sirviendo como controles mientras que el grupo B conservaba el pellet y recibía una solución de alcohol $40 \%$ disuelto en agua lo cual derivó en una esteatosis alcohólica multivesicular. Ambos grupos se mantuvieron en estas condiciones por 60 días. Posteriormente las ratas fueron sacrificadas y se extrajeron muestras tanto de hígado normal-control como de hígado graso para enseguida ser procesadas para microscopía electrónica de transmisión. De hepatocitos normales y esteatósicos se obtuvieron microfotografías electrónicas de transmisión con aumentos finales de 9.500X, las cuales fueron sometidas a estudios morfométricos para determinar fracciones volumétricas de los siguientes componentes celulares: Retículo endoplasmático rugoso, mitocondrias, inclusiones lipídicas y de glicógeno, eu y heterocromatina. De igual manera se cuantificaron las áreas celulares y nucleares. Del análisis de los resultados entre hepatocitos normales y alcohólicos se visualiza que existen notables diferencias en todos los componentes celulares cuantificados. Se concluye que los efectos de la ingesta diaria de alcohol provoca en los hepatocitos una esteatosis microvesicular que genera una drástica transformación en su ultraestructura y en su morfología, situación que se traduciría, por ende, en una variación funcional, representando de esta manera el efecto que esta droga provoca en los hepatocitos.

PALABRAS CLAVE: Esteatosis; Alcohol; Morfometría; Hepatocito.

\section{INTRODUCCIÓN}

La patología referida como hígado graso es la más frecuente de las afecciones conocidas como esteatosis, está caracterizada por la deposición anormal de lípidos tanto en los espacios intersticiales del hígado como en los citoplasmas de los hepatocitos, revelando anormalidad en los procesos de síntesis y eliminación de triglicéridos (Ishak et al., 1991).

Dichos lípidos se acumulan formando vesículas que cuando son de mayor tamaño rodean al núcleo desplazándolo hacia la periferia celular pegado a la membrana plasmática, generando así la denominada esteatosis macrovesicular. Por el contrario, si el lípido se estructura en pequeñas gotas ocupando distintas áreas del citoplasma se produce la esteatosis microvesicular (Arakawa et al. 1975).

Se ha demostrado que la etiología de la esteatosis se relaciona con componentes metabólicos y nutricionales, farmacológicos generalmente provocado por antibióticos y mayoritariamente con un consumo exacerbado de toxinas como etanol y tetracloruro de carbono (Day \& Yeaman 1994), existiendo también diversos factores de riesgo asociado a la afección tales como diabetes mellitus (Araya et al., 2006), mal nutrición proteica (Conde Martel et al., 1993), hipertensión (Brokes \& Cooper, 2007), obesidad (Saadeh, 2007) y anoxia (Cotran et al., 1998). De igual manera Day et al. (1993) sugieren intervención de factores genéticos en el desarrollo de esta patología.

Así mismo, la expresión alterada en los hepatocitos de proteínas/enzimas involucradas en la oxidación de ácidos grasos así como de las constitutivas de peroxisomas, se traduciría en la acumulación de triglicéridos hepáticos (Rehnmark et al., 1998). Por otra parte, modificaciones en la morfología tanto de la matríz como el aumento en los diámetros de la membrana interna mitocondrial, sugieren disminución en los mecanismos de beta oxidación de ácidos grasos y generación de esteatosis microvesicular (Santer et al., 1990).

\footnotetext{
" Departamento de Ciencias Básicas, Facultad de Medicina, Universidad de La Frontera, Temuco, Chile.

** Instituto de Ciencias Marinas y Limnológicas, Universidad Austral de Chile, Valdivia, Chile.

Este trabajo fue financiado por el proyecto DI-UFRO 12-0024.Universidad de La Frontera, Chile.
} 
Es objetivo fundamental del presente trabajo fue visualizar las diferencias morfológicas y de volumen que se puedan establecer mediante la microscopía electrónica de transmisión y técnicas morfométricas, comparando las fracciones volumétricas de hepatocitos normales y esteatósicos a nivel de: núcleos, citoplasmas, retículo endoplásmico rugoso, mitocondrias, inclusiones de glicógeno y gotas lipídicas. De igual manera se cuantificó la relación núcleocitoplasmática y el área nuclear y celular de ambos hepatocitos.

En la medida que se cuantifiquen y comparen a nivel ultraestructural los volúmenes que ocupan los distintos constituyentes celulares de hepatocitos normales versus los esteatósicos, se podrá determinar las variaciones en la funcionalidad que adquieren aquellos que contienen las infiltraciones lipídicas.

\section{MATERIAL Y MÉTODO}

Un total de 24 ratas hembras, de 4 meses de vida con un peso de aproximadamente 250 gramos fueron divididas en dos grupos de 12 animales cada uno rotulados como A y B. El primero se mantuvo con pellet y agua ad libitum como control, mientras que el grupo B conservaba el pellet recibiendo una solución de alcohol 40\%. Ambos grupos se mantuvieron en estas condiciones por 60 días. Posteriormente las ratas fueron sacrificadas y se extrajeron muestras tanto de hígado normal-control como hígado graso para enseguida ser procesadas para microscopía electrónica de transmisión donde a las muestras se le adicionó una solución de glutaraldehído $2 \%$ en tampón fosfato $0,15 \mathrm{M}, \mathrm{pH} 7,2$ y se mantuvo a temperatura ambiente por 2 horas. Luego, fueron sometidas a un lavado en solución de $6 \mathrm{~g}$ de $\mathrm{NaCl}$ y 73 g de sacarosa, disuelto en 1 litro de agua destilada.

La post-fijación se realizó con tetróxido de osmio 1\% disuelto en la solución antes descrita durante una hora a $40^{\circ} \mathrm{C}$ y acetato de uranilo $0,5 \%$ por 18 horas. Luego de lavado el material fue deshidratado en concentraciones crecientes de acetona (30 a 100\%) e incluido en Araldita 6005. Se obtuvieron cortes ultrafinos de aproximadamente $70 \mathrm{~nm}$ de grosor los que fueron tratados con acetato de uranilo $2 \%$ durante 40 minutos y citrato de plomo $0,5 \%$ por 10 minutos. Las muestras fueron finalmente estudiadas y microfotografiadas en un microscopio electrónico Phillips EM 300.

Análisis estadístico. A partir de los bloques para microscopia electrónica fueron obtenidos cortes ultrafinos desde los cuales se micrografiaron hepatocitos irradiados con distintas dosis con un aumento final de hasta 9.500X.
Para la evaluación de las fracciones volumétricas de los componentes celulares, fue sobrepuesto un retículo de puntos sobre las microfotografías electrónicas y se procedió al conteo diferencial de los puntos que incidían sobre los perfiles de los componentes, calculándose la fracción volumétrica que ellos ocupan, mediante la siguiente ecuación (Weibel 1969).

$$
F v=\frac{P i}{P t} \quad \begin{aligned}
& \text { Ecuación de Weibel utilizada para calcular frac- } \\
& \text { ción volumétrica de los componentes celulares. }
\end{aligned}
$$

Donde: $\mathrm{Fv}=$ Fracción volumétrica del componente celular; $\mathrm{Pi}=$ Puntos incidentes sobre el componente en estudio; y $\mathrm{Pt}=$ Puntos totales incidentes en la célula estudiada.

Todos los datos cuantitativos obtenidos fueron sometidos a la prueba de Wilcoxon para muestras no paramétricas estableciendo diferencias con un valor de $\mathrm{Z}=2,023$ y un $\mathrm{p}=0,043$ indicando entonces que existen diferencias significativas entre ellos.

\section{RESULTADOS}

La microscopía electrónica de transmisión utilizada en nuestro estudio muestra la morfología de hepatocitos normales y su contrapartida afectados con esteatosis microvesicular, detallándose en ellos la ultraestructura de sus componentes celulares, evidenciados en las Figuras 1 y 2 respectivamente y que generan los siguientes datos morfométricos los cuales se consignan en la Figura 3.

Comparándose las fracciones volumétricas correspondientes a las inclusiones lipídicas se observa con nitidéz que el volumen correspondiente al hepatocito esteatósico está aumentado en un $90 \%$.

De igual manera, cuantificándose las fracciones volumétricas de retículo endoplásmico rugoso (RER) y mitocondrias, se evidencia claramente que están mayoritariamente expresados en el hepatocito esteatósico apuntando entonces que en esta célula se realiza una notable activación de la síntesis proteica.

Igualmente en relación a las inclusiones de glicógeno cuantificadas en el hepatocito esteatósico y observándose claramente su disminución hasta un 50\%, apuntando de igual manera a una actividad celular mayor que la observada en el normal. Todos los datos anteriormente descritos están resumidos en la Figura 3. 


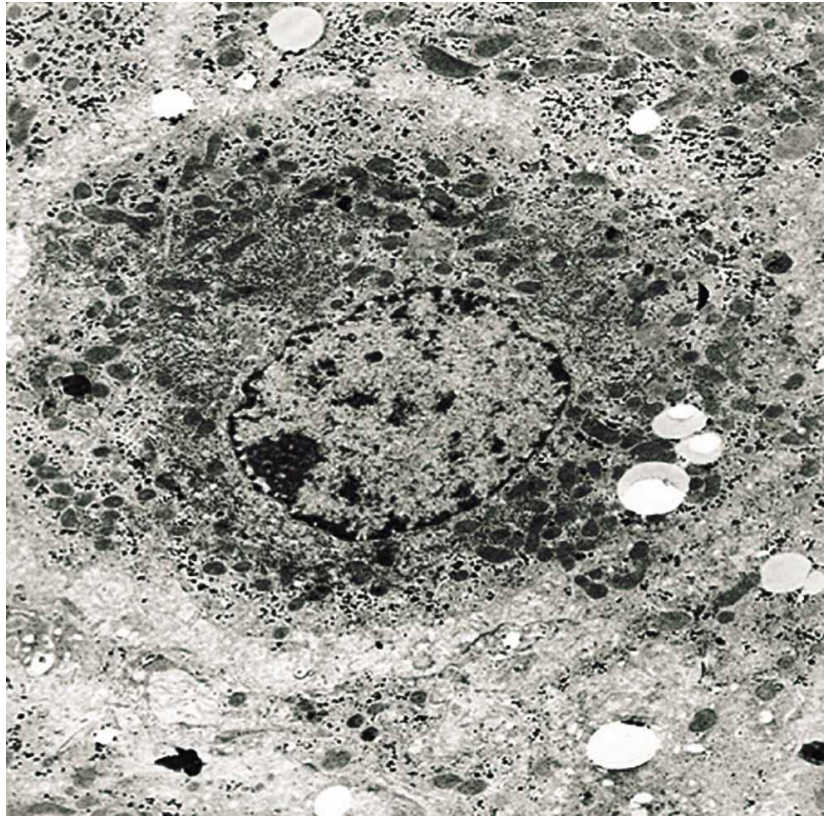

Fig. 1. Microfotografía electrónica de transmisión perteneciente a hepatocito de rata normal. 8.500X.

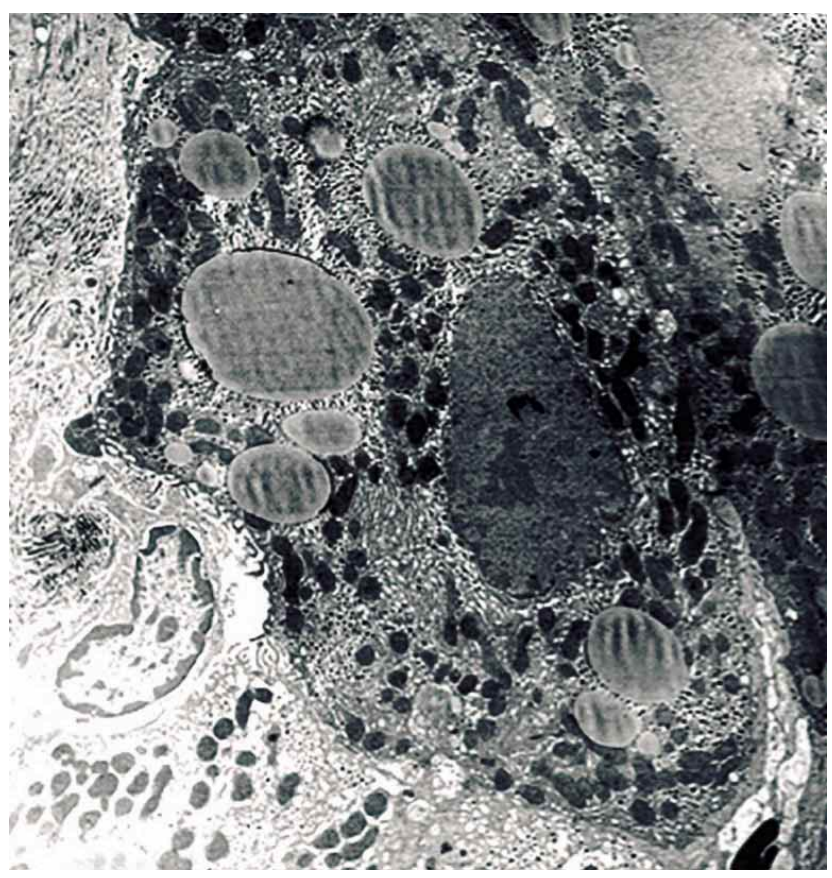

Fig. 2. Microfotografia e lectrónica de transmisión perteneciente a hepatocito de rata que presenta esteatosis microvesicular. 9.500X.

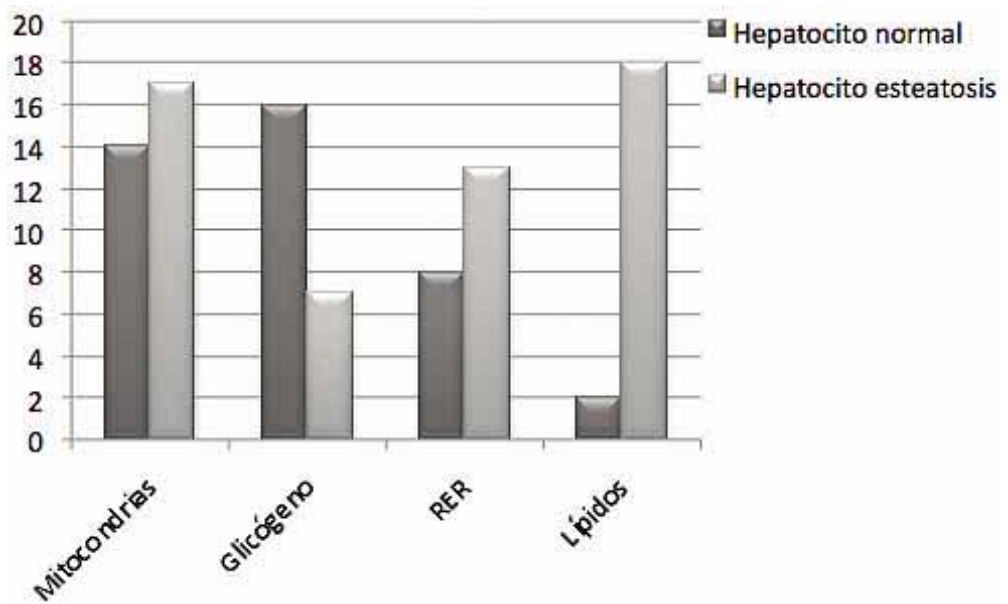

Fig. 3. Fracciones volumétricas expresadas en porcentaje correspondiente a componentes celulares pertenecientes tanto a hepatocitos normales como esteatósicos.

Tabla I. Áreas celulares y nucleares expresadas en $\mathrm{m}^{2}$ pertenecientes tanto a hepatocitos normales como esteatósicos.

\begin{tabular}{lcc}
\hline & Hepatocito Normal & Hepatocito esteatósico \\
\hline Área Celular & 248,8 & 248,5 \\
Área Nuclear & 23,4 & 27,7 \\
\hline
\end{tabular}

Tabla II. Fracciones volumétricas expresadas en porcentaje correspondiente tanto a eu como heterocromatina evaluadas en hepatocitos normales y esteatósicos.

\begin{tabular}{lcc}
\hline & Hepatocito normal & Hepatocito esteatósico \\
\hline Heterocromatina & $32 \%$ & $8 \%$ \\
Eucromatina & $68 \%$ & $92 \%$ \\
\hline
\end{tabular}

Respecto al volumen nuclear la diferencia encontrada a favor del hepatocito esteatósico equivalente a un $2 \%$ y $4,3 \mu^{2}$, se podría traducir en activos mecanismos de replicación y transcripción.

Finalmente, considerando los volúmenes cuantificados en relación a la eucromatina de hepatocitos normales respecto a su contrapartida esteatósico, se observa con claridad que la manifiesta diferencia a favor de esta última célula se traduce en una alta tasa de expresión génica y por ende, en una mayor actividad replicativa y transcripcional y guarda directa relación con los volúmenes incrementados de los componentes citosólicos descritos con anterioridad. 


\section{DISCUSIÓN}

El análisis de los resultados obtenidos evidencia que el efecto de infiltraciones lipídicas desemboca en una esteatosis microvesicular resultado de un consumo persistente de alcohol sobre hepatocitos de rata, generan notables variaciones en los componentes celulares tanto nucleares como citosólicos, las cuales provocan lógicas modificaciones a nivel morfológico, ultraestructural y de funcionalidad celular.

Nuestro hallazgo en términos de evidenciar la aparición de una esteatosis microvesicular evidenciada en la Figura 2 y caracterizada por presentar un $18 \%$ del volumen celular perteneciente a infiltraciones lipídicas provenientes de un consumo indiscriminado y diario de alcohol, es congruente con los resultados de variadas investigaciones en las cuales se genera esta afección hepática. Así Macfarlane et al. (2011) demuestran generación de esteatosis producto de deficiencia en la dieta de metionina y colina lo que provoca acumulación de triglicéridos. En este mismo sentido, Mong et al. (2011) describen que histidina, carnosita y alanina tienen acción directa sobre la expresión de RNA de enzimas lipogénicas que regulan el esterol y su deficit induciría esteatosis. Por su parte Kiefer et al. (2011) precisan que en la generación de esta afección tiene un rol fundamental el deficit de la citoquina osteopontina, la cual en situaciones normales actúa impidiendo obesidad y alteraciones hepáticas incluyendo esteatosis.

En este mismo sentido, Rehnmark et al. describen que la acumulación de triglicéridos hepáticos se debe tanto a una expresión alterada de proteínas involucradas en la oxidación de ácidos grasos, como una notable reducción en la actividad de lipasas hepáticas y otras enzimas propias del peroxisomas reducidas hasta un $60 \%$.

En este contexto y en términos generales Orlicky et al. (2011) precisan que la generación de esteatosis se debe a dos procesos que actúan sincrónicamente: un metabolismo hepático alterado o afectado asociado con un consumo crónico de alcohol.

Nuestros resultados muestran también coincidencia con lo demostrado por distintos investigadores respecto al incremento del volumen mitocondrial encontrado en los hepatocitos esteatósicos, Bezborodkina et al. (2008) describen claramente que el hepatocito alcohólico presenta un $28 \%$ de incremento en la densidad y volumen mitocondrial. Por su parte, Santer et al., informan sobre este volumen mitocondrial haciendo énfasis tanto en alteraciones de su maquinaria enzimática como también en la presencia de una matríz mitocondrial electrodensa y una hipertrofia en el diámetro de la membrana interna, todo lo cual indicaría una notable dis- minución en la oxidación de ácidos grasos. Situación semejante plantean Ruebner et al. (1969) al describir en hepatocitos esteatósicos mitocondrias con opacidad en su matriz y crestas circulares en extremo electrodensas. En contexto semejante, Tutunaru et al. (2010) han demostrado en los citoplasmas de hepatocitos con esteatosis, la presencia de mitocondrias gigantes que contienen inclusiones en sus crestas y cisternas dilatadas o hipertrofiadas pertenecientes a retículo endoplasmático rugoso.

Paralelamente, es preciso considerar que los volúmenes de glicógeno cuantificados en el hepatocito esteatósico disminuyen con respecto al normal, indicando, por ende, una constante degradación a glucosas citoplasmáticas para realizar procesos de glicólisis y posterior síntesis de ATP mitocondrial, obteniendo la energía necesaria para enfrentar mecanismos diversos, de acuerdo a nuestros resultados mostrando notables incrementos en los organelos involucrados, una activa síntesis de proteínas (Junqueira \& Salles 1975).

Este hepatocito esteatósico como ya fue mostrado en los resultados, presenta dos evaluaciones que indican una función celular mas activa que la característica de un hepatocito normal: un aumento de $4,3 \mathrm{~m}^{2}$ en su área nuclear y una fracción volumétrica de $92 \%$ perteneciente a la eucromatina, ambos valores óptimos que indicarían activos mecanismos tanto de replicación como de transcripción que determinarían una activa expresión génica y por consecuencia una acentuada síntesis proteica (Paniagua et al., 2007).

En conclusión, nuestros resultados definen con claridad las características morfométricas y ultraestructurales de la esteatosis microvesicular generada por un consumo crónico de etanol en los hepatocitos de rata, destacando que paralelamente con las infiltraciones lipídicas se ha estimulado el desarrollo de estructuras nucleares y citosólicas asociadas a síntesis proteica, en comparación con las evaluaciones de los componentes de hepatocitos normales y que, por ende, se incrementa la actividad celular en los hepatocitos esteatósicos.

CORNEJO, R.; GARRIDO, O.; SÁEZ, L.; BUSTAMANTE, C. \& CARTES, G. Morphometric analysis of alcoholic microvesicular hepatic steatosis in rats. Int. J. Morphol., 32(2):488-492, 2014.

SUMMARY: A total of 24 female rats, aged 4 months and weighing approximately $250 \mathrm{~g}$, were divided into two groups, called A and $\mathrm{B}$. The group A animals were kept on pellets and water ad libitum and served as controls, while group B animals were fed pellets and given a solution of $40 \%$ alcohol dissolved in water, leading to alcoholic multivesicular steatosis. Both groups were kept under these conditions for 60 days. The rats were then euthanized and samples of normalcontrol and fatty liver were taken and processed for examination by transmission electron microscope. Transmission electron microscope microphotographs of normal and steatotic hepatocytes were obtained 
with final magnification of $9,500 \mathrm{X}$. They were subjected to morphometric studies to determine the volumetric fractions of the following cell components: Rough Endoplasmic Reticulum, mitochondria, lipid and glycogen inclusions, and eu- and heterochromatin. In addition, the cell and nucleus areas were quantified and the nucleo cytoplasmic ratio of each cell type was determined. Analysis of the results between normal and alcoholic hepatocytes shows that noticeable differences exist in all the cell components quantified. It is concluded that the effects of the daily consumption of alcohol provoke microvesicular steatosis in the hepatocytes, generating a drastic transformation of their ultrastructure and morphology. This finally leads to functional variations, representing the effects produced by this drug in the hepatocytes.

KEY WORDS: Steatosis; Alcohol; Morphometry; Hepatocyte.

\section{REFERENCIAS}

Arakawa, M.; Taketomi, S.; Furuno, K.; Matsuo, T. \& Iwatsuka, H. Metabolic studies on the development of ethanol-induced fatty liver in KK-Ay mice. J. Nutr., 105(12):1500-8, 1975.

Araya, Q. A. V.; Valera, M. J. M.; Contreras, B. J.; Csendes, J. A.; Burdiles, P. P.; Rojas, C. J.; Maluenda, G. F.; Smok, S. G. \& Poniachik, T., J. Glucose tolerance alterations and frequency of metabolic syndrome among patients with non alcoholic fatty liver disease. Rev. Med. Chil., 134(9):1092-8, 2006.

Bezborodkina, N. N.; Okovityi, S. V.; Kudriavtseva, M. V.; Kirik, O. V.; Zarubina, I. V. \& Kudriavtsev, B. N. Morphometry of hepatocyte mitochondrial apparatus in normal and cirrhotic rat liver. Tsitologiia, 50(3):228-36, 2008

Brookes, M. J. \& Cooper, B. T. Hypertension and fatty liver: guilty by association? J. Hum. Hypertens., 21(4):264-70, 2007.

Conde Martel, A.; González Reimers, E.; Santolaria Fernández. F.; Castro Alemán, V.; Marchena Gómez, J. \& Martínez Riera, A. Liver changes in protein malnutrition. An experimental study in rats. Nutr. Hosp., 8(6):358-63, 1993.

Cotran, R.; Kumar, V. \& Robbins, S. Patologia Estrutural e Funcional. $4^{a}$ ed. Río de Janeiro, Guanabara-Kogan, 1991. pp.150.

Day, C. P.; James, O. F.; Brown, A. S.; Bennett, M. K.; Fleming, I. N. \& Yeaman, S. J. The activity of the metabolic form of hepatic phosphatidate phosphohydrolase correlates with the severity of alcoholic fatty liver in human beings. Hepatology, 18(4):832-8, 1993.

Day, C. P. \& Yeaman, S. J. The biochemistry of alcohol-induced fatty liver. Biochim. Biophys. Acta, 1215(1-2):33-48, 1994.

Ishak, K. G.; Zimmerman, H. J. \& Ray, M. B. Alcoholic liver disease: pathologic, pathogenetic and clinical aspects. Alcohol. Clin. Exp. Res., 15(1):45-66, 1991.

Junqueira, L. \& Salles, L. Ultra-estrutura e função celular. Río de Janeiro, Guanabara Koogan, 1975.
Kiefer, F. W.; Neschen, S.; Pfau, B.; Legerer, B.; Neuhofer, A.; Kahle, M.; Hrabé de Angelis, M.; Schlederer, M.; Mair, M.; Kenner, L.; Plutzky, J.; Zeyda, M. \& Stulnig, T. M. Osteopontin deficiency protects against obesity-induced hepatic steatosis and attenuates glucose production in mice. Diabetologia, 54(8):2132-42, 2011.

Macfarlane, D. P.; Zou, X.; Andrew, R.; Morton, N. M.; Livingstone, D. E.; Aucott, R. L.; Nyirenda, M. J.; Iredale, J. P. \& Walker, B. R. Metabolic pathways promoting intrahepatic fatty acid accumulation in methionine and choline deficiency: implications for the pathogenesis of steatohepatitis. Am. J. Physiol. Endocrinol. Metab., 300(2):E402-9, 2011.

Mong, M. C.; Chao, C. Y. \& Yin, M. C. Histidine and carnosine alleviated hepatic steatosis in mice consumed high saturated fat diet. Eur. $J$. Pharmacol., 653(1-3):82-8, 2011.

Orlicky, D. J.; Roede, J. R.; Bales, E.; Greenwood, C.; Greenberg, A.; Petersen, D. \& McManaman, J. L. Chronic ethanol consumption in mice alters hepatocyte lipid droplet properties. Alcohol Clin. Exp. Res., 35(6):1020-33, 2011.

Paniagua, R.; Nistal, M.; Sesma, P.; Alvarez-Uría, M.; Fraile, B.; Anadón, R. \& Sáez, F. Biología Celular. $3^{\text {a }}$ ed. Madrid, McGraw Hill, 2007.

Rehnmark, S.; Giometti, C. S.; Slavin, B. G.; Doolittle, M. \& Reue, K. The fatty liver dystrophy mutant mouse: microvesicular steatosis associated with altered expression levels of peroxisome proliferatorregulated proteins. J. Lipid Res., 39(11):2209-17, 1998.

Ruebner, B. H.; Moore, J.; Rutherford, R. B.; Seligman, A. M. \& Zuidema, G. D. Nutritional cirrhosis in rhesus monkeys: Electron microscopy and histochemistry. Exp. Mol. Pathol., 11(2):53-70, 1969.

Saadeh, S. Nonalcoholic Fatty liver disease and obesity. Nutr. Clin. Pract., 22(1):1-10, 2007.

Santer, R.; Schmidt-Sommerfeld, E.; Leung, Y. K.; Fischer, J. \& Lebenthal, E. Medium-chain acyl CoA dehydrogenase deficiency: electron microscopic differentiation from Reye syndrome. Eur. J. Pediatr., 150(2):111-4, 1990.

Tutunaru, D.; Ciocoiu, M.; Coman, M.; Musat, C.; Nechita, A. \& Badescu, M. Ethanol induced hepatic steatosis-morpho-functional aspects. Ann. Rom. Soc. Cell Biol., 15(2):362-7, 2010.

Weibel, E. R. Stereological principles for morphometry in electron microscopic cytology. Int. Rev. Cytol., 26:235-302, 1969.

\section{Dr. Ricardo Cornejo}

Facultad de Medicina

Universidad de La Frontera

Casilla 54-D

Temuco

CHILE

Email: rene.cornejo@ufrontera.cl

Recibido : 07-10-2013

Aceptado: 24-04-2014 